\title{
Microfluidic Encapsulation of Pickering Oil Microdroplets into Alginate Microgels for Lipophilic Compound Delivery
}

\author{
Marquis Melanie ${ }^{1,{ }^{*}}$, Alix Valentin ${ }^{1}$, Capron Isabelle ${ }^{1}$, Cuenot Stephane ${ }^{2}$, Zykwinska Agata ${ }^{3}$
}

1 INRA, Biopolymeres Interact Assemblages UR1268, Rue Geraudiere, F-44316 Nantes, France.

2 Univ Nantes, CNRS, Inst Mat Jean Rouxel IMN, Rue Houssiniere, F-44322 Nantes, France.

${ }^{3}$ IFREMER, Lab Ecosyst Microbiens \& Mol Marines Biotechnol, Rue lle Yeux, F-44311 Nantes, France.

*Corresponding author : Mélanie Marquis, email address : Melanie.Marquis@nantes.infra.fr

\begin{abstract}
:
Alginate microgels are widely used as delivery systems in food, cosmetics, and pharmaceutical industries for encapsulation and sustained release of hydrophilic compounds and cells. However, the encapsulation of lipophilic molecules inside these microgels remains a great challenge because of the complex oil-core matrix required. The present study describes an original two-step approach allowing the easy encapsulation of several oil microdroplets within alginate microgels. In the first step, stable oil microdroplets were formed by preparing an oil-in-water (O/W) Pickering emulsion. To stabilize this emulsion, we used two solid particles, namely the cotton cellulose nanocrystals (CNC) and calcium carbonate $(\mathrm{CaCO} 3)$. It was observed that the surface of the oil microdroplets formed was totally covered by a CNC layer, whereas $\mathrm{CaCO} 3$ particles were adsorbed onto the cellulose layer. This solid CNC shell efficiently stabilized the oil microdroplets, preventing them from undesired coalescence. In the second step, oil microdroplets resulting from the Pickering emulsion were encapsulated within alginate microgels using microfluidics. Precisely, the outermost layer of oil microdroplets composed of $\mathrm{CaCO} 3$ particles was used to initiate alginate gelation inside the microfluidic device, following the internal gelation mode. The released $\mathrm{Ca} 2+$ ions induced the gel formation through physical cross-linking with alginate molecules. This innovative and easy to carry out two-step approach was successfully developed to fabricate monodisperse alginate microgels of $85 \mathrm{pm}$ in diameter containing around 12 oil microdroplets of $15 \mathrm{mu} \mathrm{m}$ in diameter. These new oil-core alginate microgels represent an attractive system for encapsulation of lipophilic compounds such as vitamins, aroma compounds or anticancer drugs that could be applied in various domains including food, cosmetics, and medical applications.
\end{abstract}




\section{Graphical abstract}

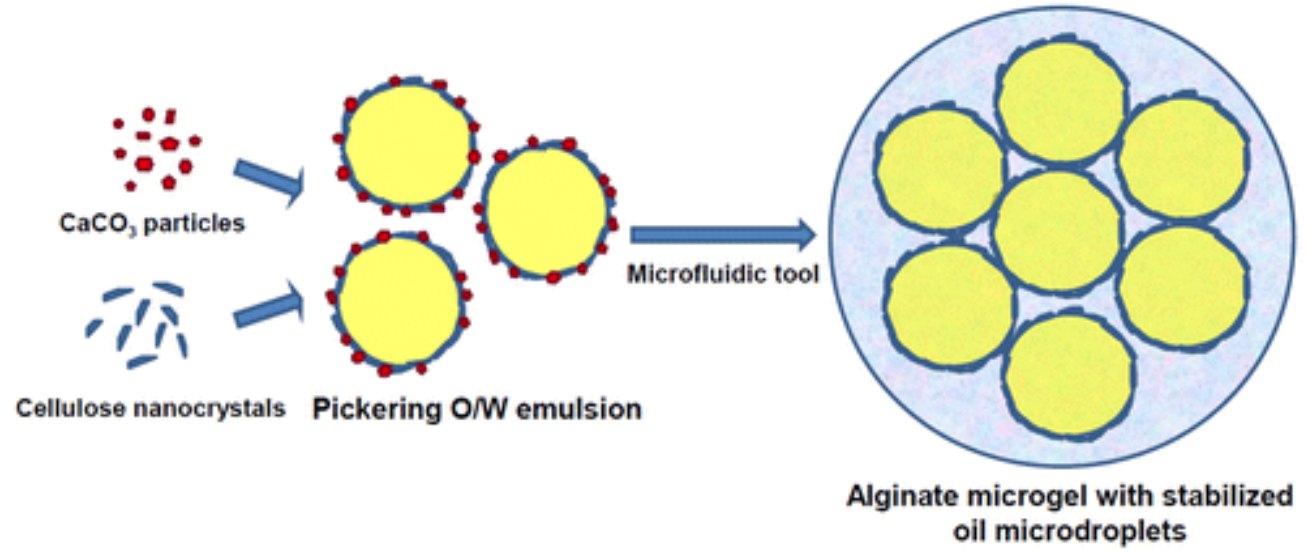

Keywords : microparticles, inonotropic gelation, calcium carbonate particles, cellulose nanocrystals, microfluidics 


\section{INTRODUCTION}

Hydrogel microparticles containing an oil core have widely been explored for their broad range of applications in food, medicine and pharmaceutics, where they are used as delivery systems for encapsulation, protection and controlled release of lipophilic compounds. ${ }^{1,2}$ Natural polymers such as polysaccharides have attracted great attention as delivery materials because of their high biocompatibility and biodegradability. Among all polysaccharides, alginate has become an excellent candidate for hydrogel microparticle formation. Due to its anionic nature, alginate can easily be structured in microgels through ionic cross-linking with divalent cations, such as calcium ions. According to the well-known "egg-box" model, ${ }^{3}$ calcium ions are implied in the formation of stable junction zones through ionic binding to pairs of carboxyl groups from two neighboring alginate chains. Alginate microgels are generally prepared following a two-step process; droplets are first generated and in the subsequent step, their gelation is induced by physical cross-linking. Micronsized droplets are usually produced by emulsification methods such as dripping, jetting, sonication as well as electrostatic droplet generation and membrane emulsification. ${ }^{4-7}$ However, none of these techniques allows to obtain droplets with a narrow size distribution and the use of both high energy and temperature may lead to alteration of the polysaccharide structure and to degradation of the biological molecules to be encapsulated within the droplets. Taking into account all these parameters, microfluidic technology, manipulating multiphase laminar flows to produce homogeneous structures, appeared as a versatile method to generate micrometer-sized droplets with controllable size and functionality. ${ }^{8-10}$ In order to induce the gelation of the droplets formed in the microfluidic device, two gelation mechanisms are mainly employed, namely external and internal gelation. In the case of external gelation, the cross-linking agent dissolved in the apolar continuous phase diffuses to the droplet phase and initiates its gelation from its surface. ${ }^{8}$ The cross-linking agent can also be dissolved in the aqueous gelling bath outside the microfluidic device, where the droplets are collected. ${ }^{11}$ However, the deformation of droplets during their transfer from the 
continuous oil phase to the gelling bath and the rapid gelation often leads to deformed microgels. The mechanism of internal gelation consists in release of the cross-linking agent dispersed in the droplet phase in its inactive form, by diffusion of an activating compound from the continuous phase into the droplets. The cross-linking agent once liberated interacts with the biopolymer molecules causing their gelation. ${ }^{8}$ Internal gelation was successfully applied to produce alginate microgels using microfluidics by integrating calcium carbonate $\left(\mathrm{CaCO}_{3}\right)$ insoluble particles into the alginate aqueous solution. ${ }^{8}$ The diffusion of acetic acid from the continuous oil phase to the droplet phase caused an increase in acidity to $\mathrm{pH}<6.5$, thus promoting $\mathrm{CaCO}_{3}$ solubilization. The liberated $\mathrm{Ca}^{2+}$ ions induced alginate chain-chain associations, which resulted in droplet gelation. ${ }^{3}$

Because of the aqueous microenvironment inside the alginate microgels, only hydrophilic compounds and cells can efficiently be encapsulated. ${ }^{12-15}$ However, in order to encapsulate lipophilic molecules, the formation of oil-core alginate microgels is required. Only few studies deal with the microfluidic fabrication of such alginate microgels that can further be used to encapsulate lipophilic compounds, thus enhancing their protection and allowing their sustained release. ${ }^{16-19}$ Ren et al. used a microfluidic approach to produce monodisperse oil-in-water-in-oil (O/W/O) double emulsions, where calcium alginate shell was formed when the alginate droplet phase encountered the $\mathrm{CaCl}_{2}$ solution in the continuous phase. ${ }^{16}$ Recently, calcium alginate microcapsules with an oil core were prepared by internal gelation of $\mathrm{O} / \mathrm{W} / \mathrm{O}$ double emulsions. The aqueous phase of the double emulsions containing alginate, $\mathrm{CaCO}_{3}$ nanoparticles and a photoacid was exposed to ultraviolet light to activate the photoacid and initiate the $\mathrm{CaCO}_{3}$ solubilization. The released $\mathrm{Ca}^{2+}$ ions induced alginate gelation. ${ }^{17} \mathrm{~A}$ microfluidic approach was recently developed to structure alginate into microfibers with encapsulated alginate particle-in-oil droplets. ${ }^{19}$ The resulting microfibers contained either a single alginate particle per oil droplet or several alginate particles per oil droplet. The multicompartment alginate fibers obtained were used to encapsulate a wide range of hydrophilic/hydrophobic and biological/inorganic compounds. Nevertheless, all these previous 
studies allowed to produce in one-step microfluidic process alginate microcapsules or microfibers containing only one oil microdroplet. Contrary to the encapsulation of only one oil droplet, the encapsulation of lipophilic compounds within several oil microdroplets should enhance their protection and ensure their sustained release.

In this context, the purpose of the present study was to develop an original two-step approach to generate monodisperse alginate microgels containing several highly stable oil microdroplets. The principal stages of the approach used are schematically presented on Figure 1. In the first step, oil microdroplets were obtained by preparing oil-in-water $(\mathrm{O} / \mathrm{W})$ Pickering emulsion, ${ }^{20}$ stabilized by solid particles, namely cotton cellulose nanocrystals $(\mathrm{CNC})$ and $\mathrm{CaCO}_{3}$. In the second step, alginate microgels containing several oil microdroplets were generated using microfluidics. The use of CNC allowed to stabilize the oil microdroplets and to prevent them from undesired coalescence phenomenon, while $\mathrm{CaCO}_{3}$ particles co-adsorbed onto the oil microdroplet surface in their inactive form triggered the gelation of alginate microdroplets formed inside the microfluidic device through in situ internal gelation mechanism. The innovative approach developed here leads to the formation of biopolymeric microgels filled with oil microdroplets that are efficiently stabilized by bio-based solid interfacial agent. The encapsulation of a model lipophilic compound, namely Nile Red dye, within oil microdroplets further loaded within alginate microgels was demonstrated.

Step 1: Preparation of O/W Pickering emulsion
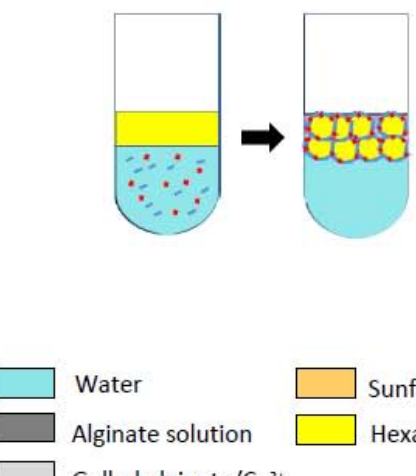

Gelled alginate/Ca ${ }^{2+}$
Step 2: Microfluidic encapsulation of O/W Pickering emulsion

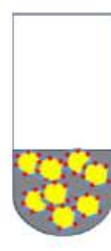

Pickering emulsion in aqueous alginate solution

$\begin{array}{ll}\square \text { Sunflower seed oil } & -\mathrm{CNC} \\ \text { Hexadecane } & -\mathrm{CaCO}_{3} \\ & \mathrm{H}+\text { Acetic acide }\end{array}$

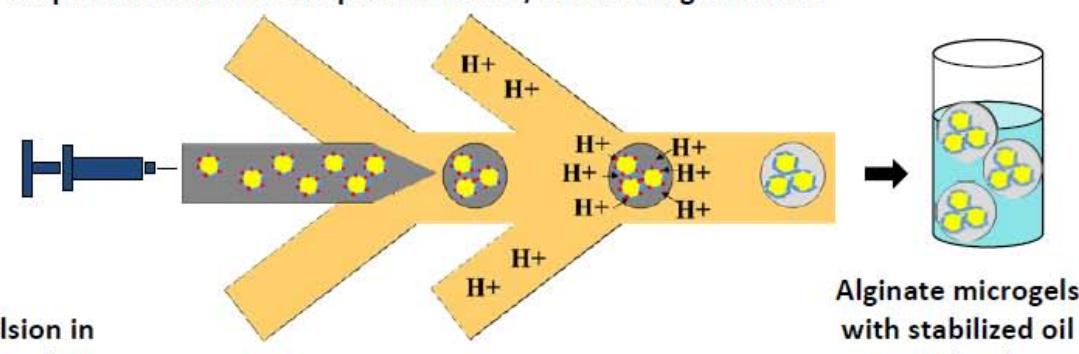

Microfluidic device microdroplets 
Figure 1. Schematic representation of the approach developed to generate monodisperse alginate microgels containing several highly stable oil microdroplets. Step 1: Preparation of an O/W Pickering emulsion stabilized by $\mathrm{CNC}$ and $\mathrm{CaCO}_{3}$. Step 2: Microfluidic encapsulation of the stabilized oil microdroplets into alginate microgels.

\section{EXPERIMENTAL SECTION}

Materials. Alginate $(\mathrm{Mw}=151550 \mathrm{~g} / \mathrm{mol}, \mathrm{Ip}=2.11)$ was obtained from FMC biopolymer (U.S.A). Hexadecane from Sigma Aldrich was purified by extensive extraction with water to remove most of the contaminating surfactants. Freeze-dried $\mathrm{CaCO}_{3}$ powder was provided by Mikhart Provençale S.A. Sunflower seed oil was purchased from Fluka. Sodium chloride (NaCl), calcium chloride $\left(\mathrm{CaCl}_{2}\right)$, Span 80 and acetic acid were purchased from Sigma-Aldrich. Water was purified with the Milli-Q reagent system (Millipore). Sulfated cotton cellulose nanocrystals (CNC) were prepared as following: $12 \mathrm{~g}$ of cellulose filter paper (Whatman grade 20Chr) were cut into small pieces and stirred overnight with $250 \mathrm{~mL}$ of distilled water. The resulting pulp, kept in an ice bath, was progressively suspended in $242 \mathrm{ml}$ of $95 \%$ sulfuric acid so as to reach a final concentration of $58 \%$ and subsequently brought to $70^{\circ} \mathrm{C}$ under stirring for $25 \mathrm{~min}$. After hydrolysis, the suspension was washed by repeated centrifugations, dialyzed to neutrality against Milli-Q water and sulfate ester groups residual counterions removed using mixed bed resin (TMD-8, Sigma). The final dispersion at a concentration of $5.8 \mathrm{~g} / \mathrm{L}$ was sonicated for $15 \mathrm{~min}(\mathrm{Q} 700$ sonicator, QSonica, Newtown, CT), filtered and stored at $4^{\circ} \mathrm{C}$.

Preparation of $\mathrm{CNC}$ and $\mathrm{CaCO}_{3}$ Particle Suspensions. CNC suspension was diluted at a concentration of $0.1 \mathrm{wt} \%$ in ultrapure water. $\mathrm{CaCO}_{3}$ particles were dispersed in ultrapure water at concentrations of $0.2 \%$ and $3 \mathrm{wt} \%$ using probe sonicator operated at an energy of $1.25 \mathrm{~W} . \mathrm{h}$ for 10 min and pulse program ( $2 \mathrm{~s}$ sonication/1 s standby) was applied. 
Preparation of Oil-in-Water (O/W) Pickering Emulsions. O/W Pickering emulsions were prepared using either free hexadecane or fluorescent hexadecane targeted with Nile Red dye and freshly sonicated aqueous suspension of (1) $\mathrm{CNC}$ at $0.1 \mathrm{wt} \%$ containing $20 \mathrm{mM} \mathrm{NaCl}$ or (2) $\mathrm{CaCO}_{3}$ at $0.2 \mathrm{wt} \%$ and $\mathrm{CNC}$ at $0.1 \mathrm{wt} \%$ containing $20 \mathrm{mM} \mathrm{NaCl}$. For the preparation of fluorescent hexadecane, Nile Red powder was solubilized in a volume of hexadecane by ultrasonic treatment, and the excess of no solubilized dye was eliminated by centrifugation. $\mathrm{O} / \mathrm{W}$ ratio was fixed at 20/80. Practically, $0.6 \mathrm{~mL}$ of hexadecane were added to $2.4 \mathrm{~mL}$ of an aqueous nanoparticle suspension in a plastic vial and sonicated. The energy of 0.047 W.h was used and applied by alternating $2 \mathrm{~s}$ sonication with a $1 \mathrm{~s}$ standby for $1 \mathrm{~min}$.

Microfluidic Device. A microfluidic flow-focusing device with three inlets (Figure S1 A) was fabricated by soft lithography technique using poly(dimethylsiloxane) (PDMS) (RTV 615, Elecoproduit, France). ${ }^{21}$ SU-8 (CTS, France) positive relief structures were produced on silicon wafers. PDMS polymer (in a mixture of 10:1 base polymer:curing agent) was cast from this mould, and access holes were punched on the PDMS layer. The PDMS layer (in a mixture of 10:1 base polymer:curing agent) was then placed in contact with a thin PDMS layer (in a mixture of 20:1 base polymer:curing agent) to generate the microchip. The cross-linker diffused as a result of the gradient from PDMS (10:1) to PDMS (20:1). The chip was then oven-treated at $70^{\circ} \mathrm{C}$ for $24 \mathrm{~h}$ to strengthen the cross-linking. The designed microchannels were rectangular in shape with a uniform height of $130 \mu \mathrm{m}$. The width of all channels was of $150 \mu \mathrm{m}$, with the exception of the restriction of $100 \mu \mathrm{m}$ and the central channel of $200 \mu \mathrm{m}$ in width. The length of the central channel was of 20 mm. All dimensions were determined by profilometry and are presented on the Figure S1 A (Supporting Information). The outlet at the end of the PDMS microdevice was parallel to the central channel and a polytetrafluoroethylene (PTFE) tube (inner diameter, $0.3 \mathrm{~mm}$; outer diameter, 0.76 $\mathrm{mm}$; length, $20 \mathrm{~cm}$ ) was directly inserted into a PDMS short exit channel (depth, $130 \mu \mathrm{m}$; width, $400 \mu \mathrm{m}$; length, $5 \mathrm{~mm}$ ). 


\section{Fabrication of Alginate Microgels Containing O/W Pickering Emulsion Using}

Microfluidics. Microfluidic device containing three inlets was designed to produce the alginate microdroplets at the first junction: inlet 1 (I1) delivering the dispersed phase composed of a Pickering $\mathrm{O} / \mathrm{W}$ emulsion stabilized with $\mathrm{CNC}$ at $0.1 \mathrm{wt} \%, \mathrm{NaCl}$ at $20 \mathrm{mM}$ and $\mathrm{CaCO}_{3}$ at $0.2 \mathrm{wt} \%$, mixed $(1: 1, \mathrm{v} / \mathrm{v})$ with sodium alginate aqueous solution prepared at a concentration of $2 \mathrm{wt} \%$ and inlet 2 (I2) for the continuous phase, a sunflower seed oil (Fluka) with Span 80 at $1 \mathrm{wt} \%$. Inlet 3 (I3) delivering sunflower seed oil mixed with Span $80(1 \mathrm{wt} \%)$ and acetic acid $(0.5 \mathrm{wt} \%)$ at the second junction, allowed to induce the alginate droplet gelation (Figure S1 B in Supporting Information). Teflon tubing connected the syringes with the inlets of the device and the outlet of the device with a tube containing $\mathrm{CaCl}_{2}$ solution at $1 \mathrm{wt} \%$. Digitally controlled syringe pumps (Harvard Apparatus PHD 2000) delivered all liquid phases to the microfluidic device. The following flow rates of the dispersed phase: alginate aqueous solution containing the Pickering emulsion at 1 $\mu \mathrm{L} / \mathrm{min}$ and of the continuous phase: oil at $19 \mu \mathrm{L} / \mathrm{min}$ were applied during the experiment. The microgel suspension was washed three times with water and stored in $\mathrm{CaCl}_{2}$ solution at $1 \mathrm{wt} \%$ at $4^{\circ} \mathrm{C}$ for further studies.

Degradation of Alginate Microgels Loaded with the O/W Pickering Emulsion. The degradation was performed by incubating the suspension of alginate microgels loaded with fluorescent oil microdroplets either in $55 \mathrm{mM}$ sodium citrate or in $50 \%$ (v/v) ethanol for $1 \mathrm{~h}$.

Characterization Methods. Fluorescence and phase contrast microscopy images were captured with an Olympus IX51 inverse microscope (Olympus, France) equipped with a phase contrast illumination, a standard red filter (BP 510-550 nm, DM $570 \mathrm{~nm}$, BA $590 \mathrm{~nm}$ ) and a digital camera (Hamamatsu, France). The number of oil microdroplets encapsulated into fifty alginate microgels was counted from optical microscopy images. Droplet diameters of both the Pickering emulsions and alginate microgels were measured using the ImageJ freeware v1.35c. Zeta potential was measured in aqueous suspensions of $\mathrm{CaCO}_{3}$ at $0.1 \mathrm{wt} \%, \mathrm{CNC}$ at $0.1 \mathrm{wt} \%$ and $\mathrm{CNC}$ at $0.1 \mathrm{wt} \%$ 
with $20 \mathrm{mM} \mathrm{NaCl}$ using Malvern 3000 ZetasizerNanoZS (Malvern Instruments, U.K.). Scanning electron microscopy (SEM) observations were made using JEOL 7600F instrument (JEOL, US). Pickering emulsions for SEM were prepared as previously described ${ }^{22}$ from styrene/water emulsions stabilized with (1) $\mathrm{CNC}$ at $0.1 \mathrm{wt} \%$ with $20 \mathrm{mM} \mathrm{NaCl}$ or (2) $\mathrm{CNC}$ at $0.1 \mathrm{wt} \%, 20 \mathrm{mM}$ $\mathrm{NaCl}$ and $\mathrm{CaCO}_{3}$ at $0.2 \mathrm{wt} \%$. Dried beads were metalized with platinum prior to SEM observations. AFM images of dried particle suspensions and styrene/water Pickering emulsions deposited onto freshly cleaved mica surface were recorded with a NanoWizard ${ }^{\circledR}$ Atomic Force Microscope (JPK, Germany) operating in intermittent contact mode under ambient conditions. A standard rectangular cantilever (Nanosensors NCL-W) was used for imaging, with a free resonance frequency of 165 $\mathrm{kHz}$ and a typical spring constant of about $40 \mathrm{~N} / \mathrm{m}$. The radius curvature of the tip was $\sim 10 \mathrm{~nm}$. In order to check the reproducibility of the observed morphology, all samples were scanned at least on three different zones. Each sample was investigated using fresh tips previously cleaned by UVozone treatment. The length and height measurements were done using JPK Data Processing software (JPK, Germany).

\section{RESULTS AND DISCUSSION}

Preparation of $\mathbf{O} / \mathbf{W}$ Pickering Emulsion. In the present study, monodisperse alginate microgels encapsulating several highly stable oil microdroplets were generated using an original two-step approach (Figure 1). In the first step, oil microdroplets were produced by preparing an O/W Pickering emulsion. Pickering emulsions require an interfacial solid material that exhibits affinity for the two phases of the emulsion..$^{23,24}$ Therefore, an amphiphilic character of the solid emulsifier is necessary to ensure its efficient adsorption at the liquid interface. Particles with a more hydrophilic character lead to $\mathrm{O} / \mathrm{W}$, whereas more hydrophobic particles lead to inverse $\mathrm{W} / \mathrm{O}$ emulsions. ${ }^{23,24}$ The irreversible adsorption of solid particles at the liquid interface allows not only to stabilize the emulsion droplets against coalescence but also to create a physical barrier for 
material transfer. ${ }^{25}$ Recently, solid cellulosic nanocrystals were shown to be excellent candidates to stabilize O/W Pickering emulsions. ${ }^{26-28}$ Cellulosic nanocrystals used in the present study were obtained from cotton linters by a standard procedure involving sulfuric acid. ${ }^{29,30}$ The use of concentrated sulfuric acid allows the hydrolysis of the native cellulose microfibrils and removal of the amorphous or less ordered regions. In addition, during hydrolysis, some negatively charged sulfate groups are introduced by esterification of hydroxyl groups, which leads to sulfated nanocrystals. The presence of negatively charged sulfate groups allows the formation of a stable colloidal suspension. Typical AFM image of dried CNC suspension is presented on Figure 2. CNC nanorods of an average length of $190 \mathrm{~nm} \pm 20 \mathrm{~nm}$ and an average height of $9.4 \mathrm{~nm} \pm 2.4 \mathrm{~nm}$ were observed $(\mathrm{N}=50)$.
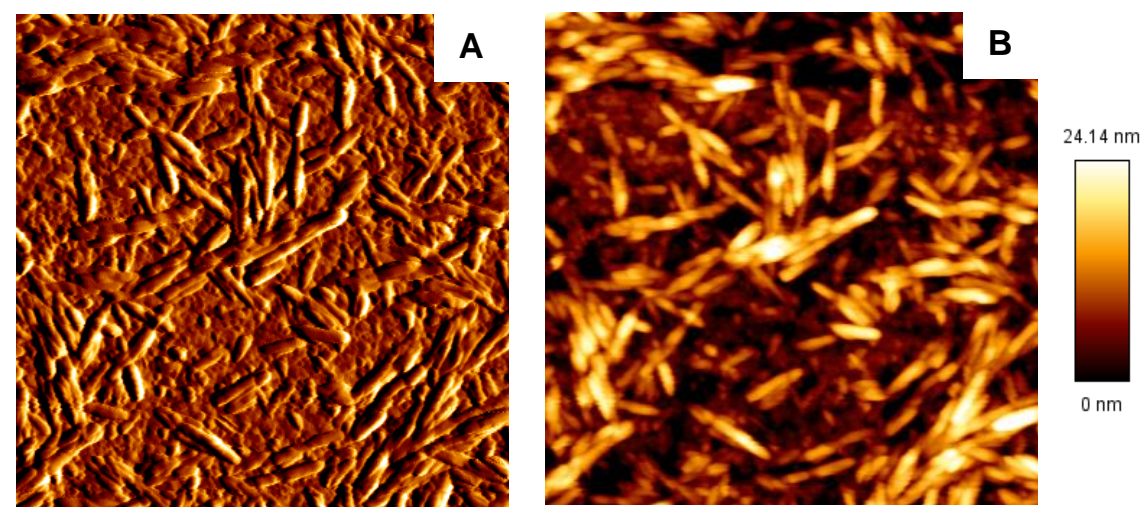

Figure 2. AFM (A) amplitude and (B) height images of dried aqueous suspension of CNC nanorods $(2 \mu \mathrm{m} \times 2 \mu \mathrm{m})$.

It was previously shown that in order to obtain a stable O/W Pickering emulsion, the repulsive interactions between highly charged $\mathrm{CNC}$, which limit their adsorption at the interface, should be screened by addition of salt. ${ }^{27}$ The addition of $20 \mathrm{mM} \mathrm{NaCl}$ was essential to efficiently increase the zeta potential of $\mathrm{CNC}$ suspension and to obtain an optimum emulsion volume. ${ }^{27}$ In agreement with these previous results, the zeta potential of an aqueous $\mathrm{CNC}$ suspension at $0.1 \mathrm{wt} \%$ measured in the absence of salt was negative with a value of $-53 \mathrm{mV}$, whereas the addition of $20 \mathrm{mM} \mathrm{NaCl}$ caused an increase in the zeta potential up to $-33 \mathrm{mV}$. Figure $3 \mathrm{~A}$ presents a typical hexadecane-in-water 
Pickering emulsion obtained and stabilized by $\mathrm{CNC}$ at $0.1 \mathrm{wt} \%$ and $20 \mathrm{mM} \mathrm{NaCl}$. As hexadecane has a lower density than water $\left(\mathrm{d}=0.82 \mathrm{~g} / \mathrm{cm}^{3}\right.$ at $\left.25^{\circ} \mathrm{C}\right)$, a creaming process is always observed. The droplet size of the Pickering emulsion, as assessed by optical microscopy (Figure 3B), was slightly polydisperse with an average diameter of $11 \mu \mathrm{m} \pm 2 \mu \mathrm{m}(\mathrm{N}=50)$.

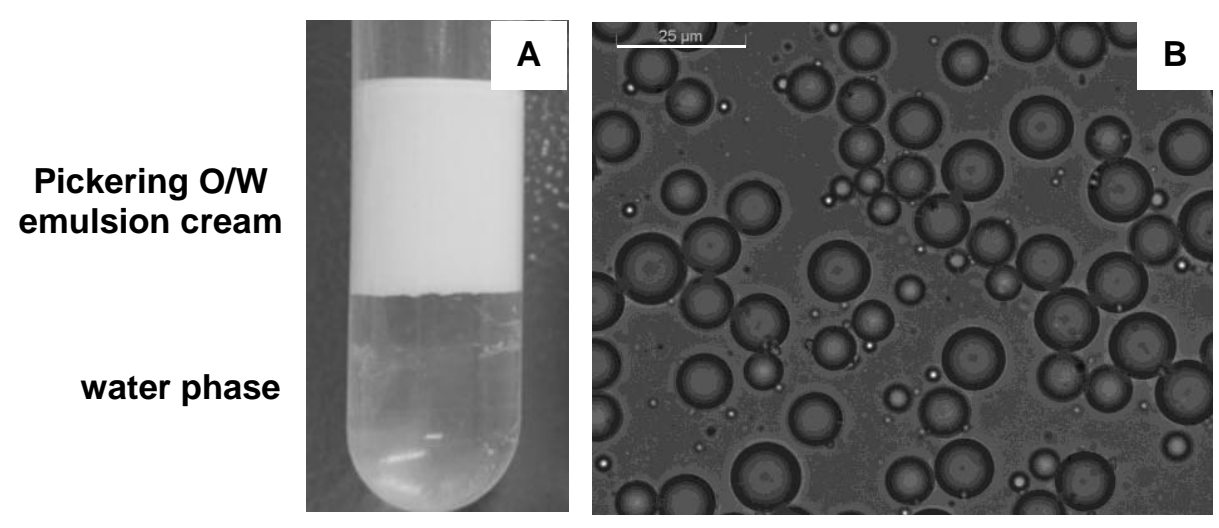

Figure 3. (A) Typical $\mathrm{O} / \mathrm{W}$ Pickering emulsion obtained with hexadecane and an aqueous suspension of $\mathrm{CNC}$ at $0.1 \mathrm{wt} \%$ and $20 \mathrm{mM} \mathrm{NaCl}$. (B) Optical image of the $\mathrm{O} / \mathrm{W}$ Pickering microdroplets obtained (scale bar of $25 \mu \mathrm{m}$ ).

The surface morphology of stabilized oil microdroplets formed was examined by SEM and AFM. Since hexadecane-in-water emulsions can not be depicted by the classical SEM approach, polymerizable resins offer a good alternative for sample preparation prior to SEM observations. Because the surface tensions of hexadecane and styrene are very similar $(27$ and $32 \mathrm{mN} / \mathrm{m}$, respectively), their ability to produce stable Pickering emulsions was shown to be similar. ${ }^{22,27}$ SEM and AFM observations were then possible after styrene polymerization. Figure 4A presents a SEM image of a polymerized styrene microdroplet stabilized by CNC. By focusing on the microdroplet surface, SEM and AFM images clearly revealed that the surface was fully covered by a layer of cellulose nanocrystals, which formed a dense solid shell (Figures 4B-C). The ability of rather 
hydrophilic $\mathrm{CNC}$ to stabilize $\mathrm{O} / \mathrm{W}$ emulsion is related to the fact that the nanocrystals expose their less polar groups at the interface, thus allowing the interaction with the oil phase. ${ }^{27,31,32}$
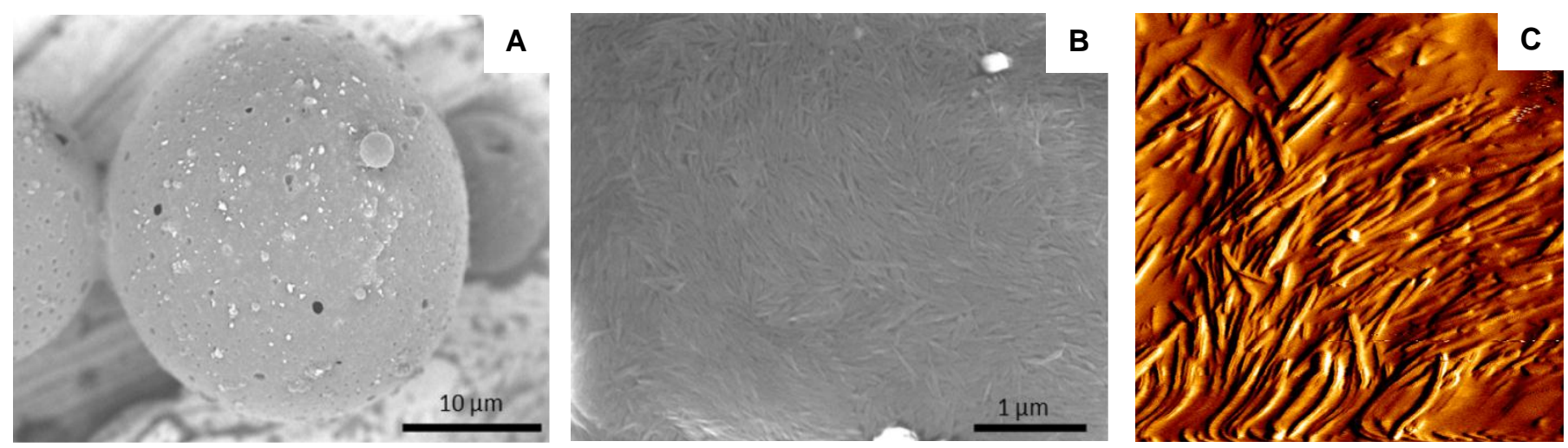

Figure 4. (A) SEM image of a styrene microdroplet stabilized by CNC at $0.1 \mathrm{wt} \%$. (B) SEM and (C) AFM amplitude images (1 $\mu \mathrm{m} \times 1 \mu \mathrm{m})$, showing the CNC layer covering the microdroplet surface.

The aim of this first step was to obtain a stable O/W Pickering emulsion stabilized by $\mathrm{CNC}$, which additionally contains $\mathrm{CaCO}_{3}$ particles available for induction of alginate gelation. Therefore, in the subsequent step, the emulsion was prepared by mixing hexadecane with an aqueous suspension of $\mathrm{CNC}$ at $0.1 \mathrm{wt} \%, 20 \mathrm{mM} \mathrm{NaCl}$ and $\mathrm{CaCO}_{3}$ particles at $0.2 \mathrm{wt} \% . \mathrm{CaCO}_{3}$ particles are strongly hydrophilic and they have been shown to stabilize O/W Pickering emulsions after modification of their wettability by interaction with amphiphiles, such as sodium dodecyl sulfate. ${ }^{33}$ Recently, O/W Pickering emulsions were also stabilized by unmodified $\mathrm{CaCO}_{3}$ nanoparticles. ${ }^{34}$ However, the interfacial properties of $\mathrm{CaCO}_{3}$ particles in the presence of $\mathrm{CNC}$ have never been examined before. AFM image of dried aqueous $\mathrm{CaCO}_{3}$ suspension presented on Figure 5 shows an important polydispersity in the particle size. Indeed, small spherical particles of an average diameter of $90 \mathrm{~nm}$ $\pm 15 \mathrm{~nm}$ were observed together with large particles of $220 \mathrm{~nm} \pm 34 \mathrm{~nm}$ and particle aggregates of $525 \mathrm{~nm} \pm 36 \mathrm{~nm}$ in length $(\mathrm{N}=20)$. Figure 6 presents a typical hexadecane-in-water Pickering 
emulsion stabilized by $\mathrm{CNC}$ at $0.1 \mathrm{wt} \%, 20 \mathrm{mM} \mathrm{NaCl}$ and $\mathrm{CaCO}_{3}$ at $0.2 \mathrm{wt} \%$. An average diameter of $15 \mu \mathrm{m} \pm 2 \mu \mathrm{m}(\mathrm{N}=50)$ was measured for oil microdroplets formed in the presence of CNC and $\mathrm{CaCO}_{3}$ particles, which is slightly higher than the droplet diameter obtained with $\mathrm{CNC}$ alone (11 $\mu \mathrm{m} \pm 2 \mu \mathrm{m})$. It can be thought that the presence of $\mathrm{CaCO}_{3}$ particles disturbed the adsorption of CNC onto the oil surface, which led to formation of oil microdroplets with slightly higher diameters.
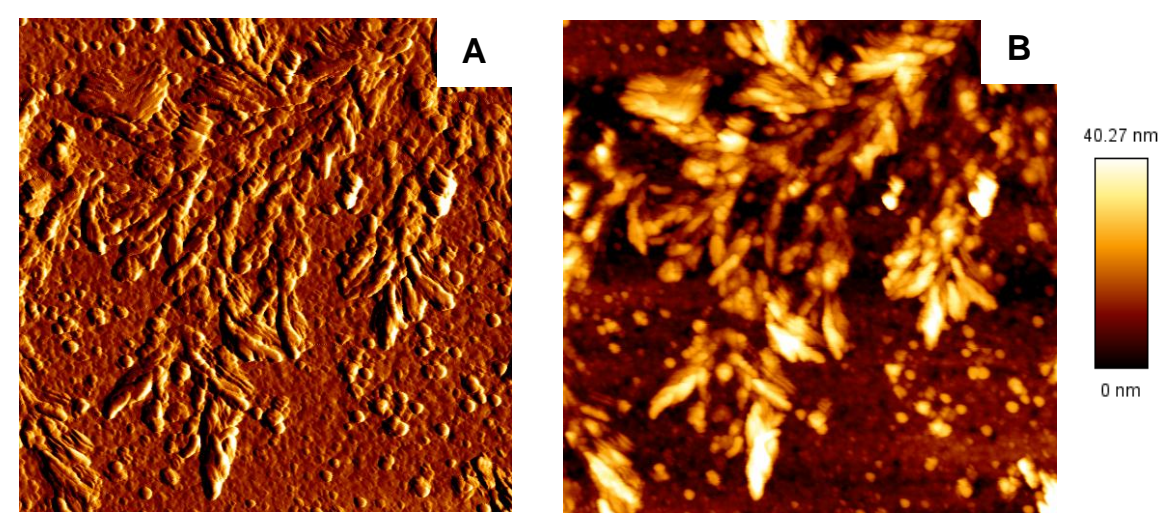

Figure 5. AFM (A) amplitude and (B) height images of dried aqueous suspension of $\mathrm{CaCO}_{3}$ particles $(3 \mu \mathrm{m} \times 3 \mu \mathrm{m})$.

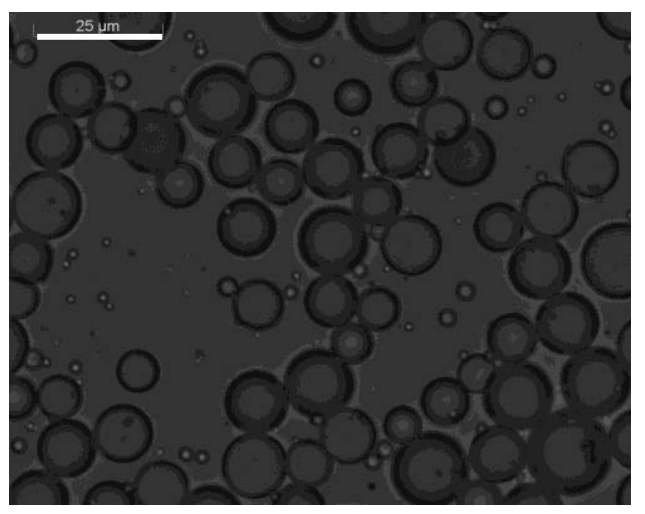

Figure 6. Optical image of the $\mathrm{O} / \mathrm{W}$ Pickering microdroplets stabilized by $\mathrm{CNC}$ at $0.1 \mathrm{wt} \%, 20 \mathrm{mM}$ $\mathrm{NaCl}$ and $\mathrm{CaCO}_{3}$ at $0.2 \mathrm{wt} \%$ (scale bar of $25 \mu \mathrm{m}$ ). 
In order to visualize the surface morphology of the O/W Pickering emulsion obtained with the mixture of $\mathrm{CNC}$ and $\mathrm{CaCO}_{3}$ particles, SEM and AFM observations were made on styrene-in-water emulsions. $\mathrm{CaCO}_{3}$ particles were clearly visible on the surface of a polymerized styrene microdroplet (Figure 7A). Further observations allowed to determine that the whole microdroplet surface is totally covered by $\mathrm{CNC}$ with $\mathrm{CaCO}_{3}$ particles adsorbed onto the $\mathrm{CNC}$ layer (Figure $7 \mathrm{~B}$ and C). Although small $\mathrm{CaCO}_{3}$ crystals of an average size of $200 \mathrm{~nm}$, previously observed on AFM images on Figure 5, were predominantly adsorbed, several larger aggregates were also noticed on the CNC surface. AFM image additionally revealed that CNC were also adsorbed onto the surface of large $\mathrm{CaCO}_{3}$ particles (Figure $7 \mathrm{C}$ ). Because the presence of $\mathrm{NaCl}$ is required during the preparation of the $\mathrm{O} / \mathrm{W}$ Pickering emulsion stabilized by $\mathrm{CNC}$, it can not be excluded that some $\mathrm{NaCl}$ particles crystallized with $\mathrm{CaCO}_{3}$ and $\mathrm{CNC}$ particles.

The particular organization observed on the surface of oil microdroplets results most likely from the higher ability of the $\mathrm{CNC}$ to stabilize the oil/water interface due to their amphiphilic nature in comparison to hydrophilic $\mathrm{CaCO}_{3}$ particles. As $\mathrm{CNC}$ expose their hydrophobic plane toward the oil phase, their hydrophilic planes with hydroxyl groups might in turn be exposed into the water. Weak hydrophilic interactions can therefore be responsible for both the adsorption of $\mathrm{CaCO}_{3}$ particles onto the $\mathrm{CNC}$ layer and $\mathrm{CNC}$ adsorption onto the $\mathrm{CaCO}_{3}$ particle surface, especially if calcium atoms are hydroxylated, ${ }^{33}$ i.e. they form $\mathrm{Ca}-\mathrm{OH}$ groups in water.
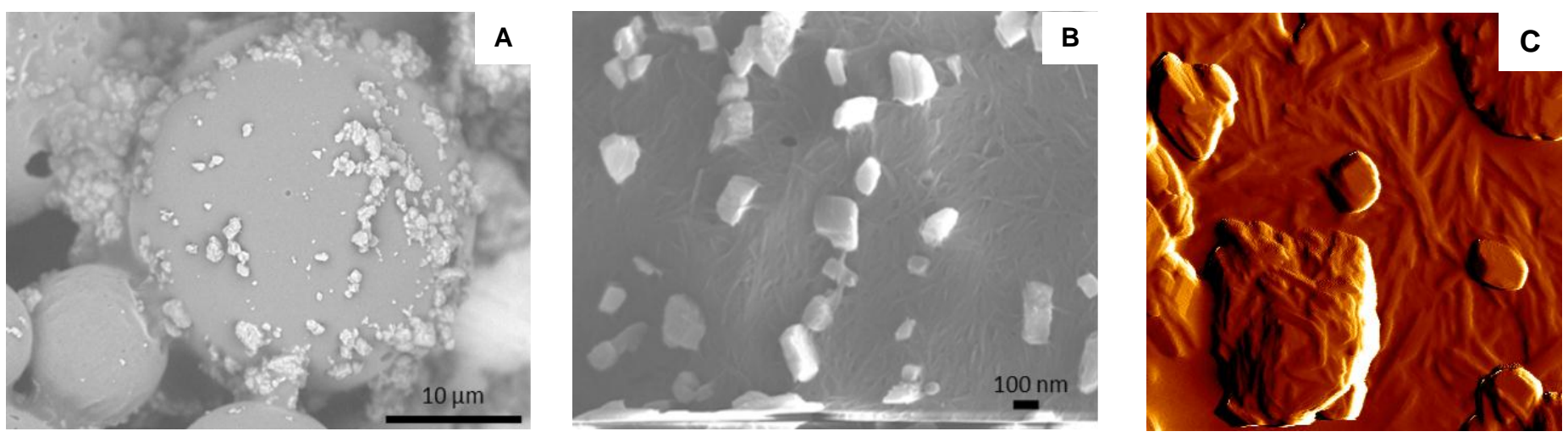
Figure 7. (A) SEM image of a styrene microdroplet stabilized by $\mathrm{CNC}$ at $0.1 \mathrm{wt} \%$ and $\mathrm{CaCO}_{3}$ at 0.2 wt\%. (B) SEM and (C) AFM amplitude images $(1 \mu \mathrm{m} \times 1 \mu \mathrm{m})$, showing the $\mathrm{CaCO}_{3}$ particles adsorbed onto the CNC layer.

\section{Fabrication of Alginate Microgels Containing O/W Pickering Emulsion using}

Microfluidics. In the second step of the present approach, oil microdroplets of $15 \mu \mathrm{m} \pm 2 \mu \mathrm{m}$ in diameter stabilized by $\mathrm{CNC}$ and $\mathrm{CaCO}_{3}$ were integrated into alginate microdroplets generated by microfluidics. The flow-focusing geometry of the microfluidic device used to structure alginate solution mixed with the $\mathrm{O} / \mathrm{W}$ Pickering emulsion into microgels is presented on Figure 8 (A and $\mathrm{B}$ ). Alginate microdroplets were formed at the first junction, while their gelation was induced at the second junction. The addition of acetic acid into the oil phase at the second junction allowed to initiate the gel formation following the internal gelation mode. The diffusion of acetic acid from the continuous phase into the aqueous droplets leads to a decrease in $\mathrm{pH}$, which results in solubilization of $\mathrm{CaCO}_{3}$ solid particles adsorbed onto the surface of oil microdroplets from the Pickering emulsion. The fact that $\mathrm{CaCO}_{3}$ particles are directly adsorbed onto the oil microdroplet surface initiates the gelation of alginate molecules at the microdroplet surface through physical crosslinking with released $\mathrm{Ca}^{2+}$ ions. The stability of oil droplets is thus enhanced. The average diameter of alginate microgels formed was of $85 \mu \mathrm{m} \pm 4 \mu \mathrm{m}(\mathrm{N}=50)$ (Figure $8 \mathrm{C}$ and $\mathrm{D}$ ). Since the surface of oil microdroplets is efficiently stabilized by a CNC layer surrounded by a gelled alginate layer, these emulsified droplets remain stable several months when stored in $\mathrm{CaCl}_{2}$ solution and no coalescence was observed inside the alginate microgels during this time. In fact, the average diameter of oil microdroplets inside the microgels $(16 \mu \mathrm{m} \pm 3 \mu \mathrm{m})$ was very close to that measured on the initial Pickering emulsion $(15 \mu \mathrm{m} \pm 2 \mu \mathrm{m})$. From fifty formed microgels, the average number of encapsulated oil cores was $12 \pm 3$. The weak variation of this number between different microgels is directly related to the small variation in size of the oil microdroplets. 
In future, the size of the alginate microgel droplet could be controlled by taking advantages of the microfluidic technique. This control offers a way to adjust the number of encapsulated oil microdroplets depending on the aimed application. Indeed, the microgel droplet size can be tuned by playing with $\mathrm{Ca}$ and $Q$ values. $^{10,35,36} \mathrm{Ca}$ being defined by the product of the viscosity and the velocity of the continuous phase divided by the interfacial tension between the two phases and the flow ratio, $Q$, corresponds to the ratio between the flow rates of the continuous phase to the dispersed one.
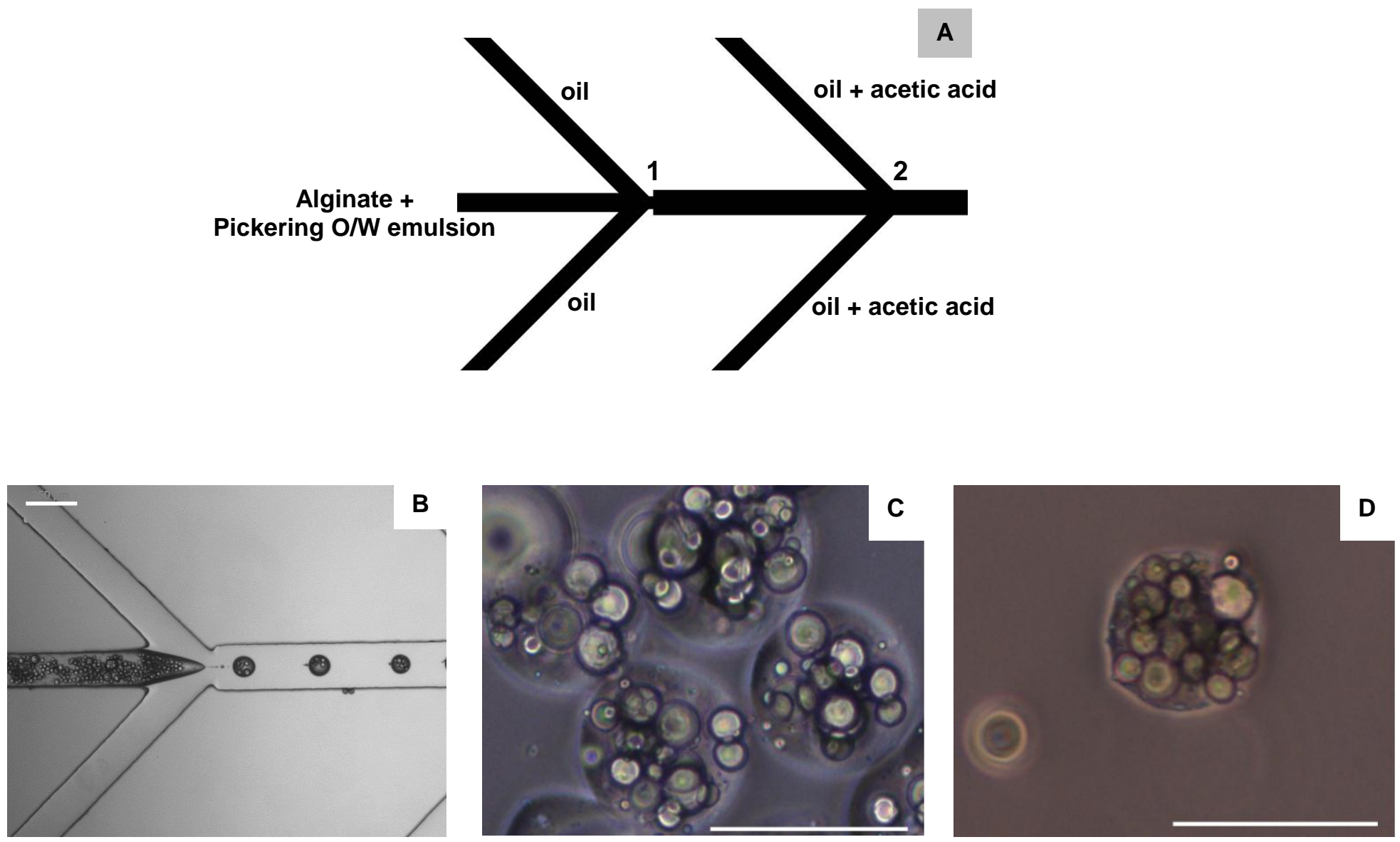

Figure 8. (A) Schematic representation of the microfluidic flow-focusing geometry used to generate alginate microgels filled with the $\mathrm{O} / \mathrm{W}$ Pickering emulsion. Alginate microdroplets were formed at the first junction (1), whereas their gelation was induced at the second junction (2), following the internal gelation mode using acetic acid as activating agent. (B) Microfluidic production of alginate 
microdroplets containing the $\mathrm{O} / \mathrm{W}$ Pickering emulsion (scale bar of $250 \mu \mathrm{m}$ ). (C-D) Alginate microgels with oil microdroplets collected in a $\mathrm{CaCl}_{2}$ bath (scale bar of $100 \mu \mathrm{m}$ ).

Encapsulation of a Lipophilic Model Compound and its Release. In order to demonstrate that the two-step approach developed in the present study could be easily applied to encapsulate lipophilic compounds, a model lipophilic molecule, Nile Red fluorescent dye was used. Nile Red, 9diethylamino-5-benzo[ $\alpha]$ phenoxazine-5-one, is a hydrophobic molecule widely used as a lipid probe. ${ }^{19,37}$ This fluorescent dye dissolves easily in a wide range of organic solvents, but negligibly in water. Nile Red dye was thus solubilized in hexadecane prior to prepare the $\mathrm{O} / \mathrm{W}$ Pickering emulsion stabilized by $\mathrm{CNC}$ and $\mathrm{CaCO}_{3}$. The obtained Pickering emulsion was then encapsulated within alginate microgels generated by microfluidics, as previously shown on Figure 8 . The encapsulation of Nile Red dye into oil microdroplets and their successful incorporation within alginate microgels was followed by fluorescence microscopy. Figure 9A presents alginate microgels loaded with fluorescent oil microdroplets, which remained highly stable when stored in $\mathrm{CaCl}_{2}$ solution and no coalescence of the oil microdroplets was observed. No diffusion of a lipophilic dye outside the oil microdroplets was evidenced even after several days following the encapsulation. This suggests that a solid shell formed by adsorbed CNC efficiently protects the oil microdroplets and their content.

Oil microdroplets loaded with the fluorescent dye can be released from alginate microgels upon different conditions. In the present study, the degradation of alginate microgels loaded with fluorescent oil microdroplets was studied in two aqueous solutions containing either sodium citrate or ethanol. Due to chelating properties of sodium citrate, calcium cations are removed from alginate microgels, thereby uncrosslinking the gel. As observed on Figure 9B, already after few minutes, the alginate matrix started to dissolve and the oil microdroplets, previously encapsulated, were progressively surrounded by the solvent. All these microdroplets were completely released in the 
aqueous solution of sodium citrate after $1 \mathrm{~h}$. Moreover, several days after the alginate microgel degradation, the released oil microdroplets remained fluorescent, which emphasizes their high stability arising most likely from the CNC solid shell. In contrast, the addition of ethanol completely destructured alginate microgels and caused the coalescence of the encapsulated oil microdroplets (Figure 9C). In fact, the addition of solution miscible with the continuous phase was previously shown to destabilize the Pickering microdroplets dispersed in the continuous phase, which led to their coalescence. ${ }^{38}$ As hexadecane used as oil phase for the Pickering emulsion preparation is slightly miscible with ethanol, ${ }^{39}$ it diffused from the oil microdroplets into the suspending solution, which led to the phase separation phenomenon. Indeed, already after $1 \mathrm{~h}$ of incubation in ethanol, fluorescent oil started to be clearly visible outside the alginate matrix. The degradation experiments performed put forward that the oil microdroplets can be released from alginate microgels upon demand and their content can be liberated using appropriate solvents. 


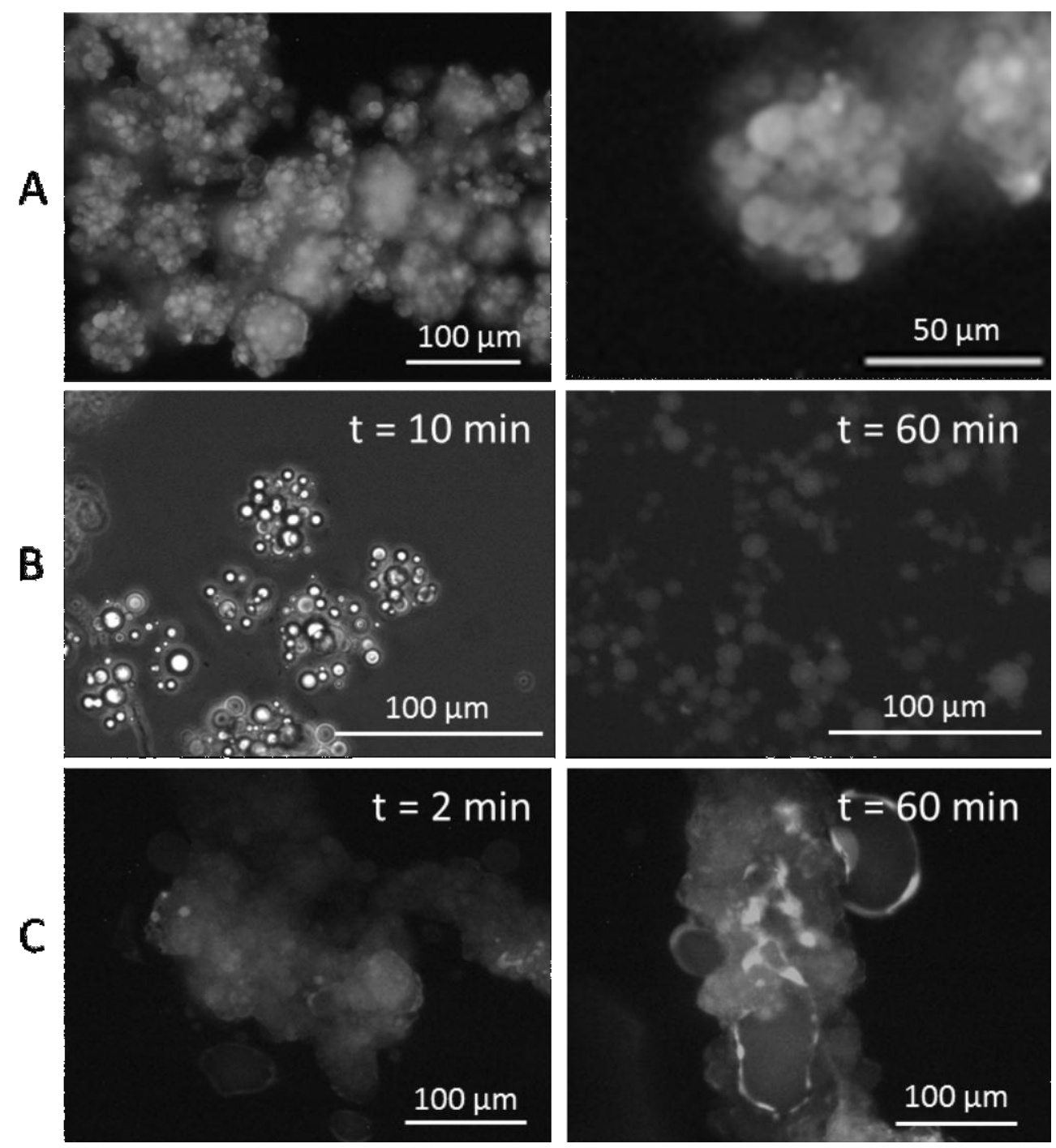

Figure 9. (A) Fluorescence optical microscopy images of alginate microgels containing highly stable Nile Red fluorescent dye loaded oil microdroplets. (B) Alginate microgels upon incubation in aqueous solution of sodium citrate. Images obtained by phase contrast coupling with fluorescent optical microscopy on the left and by fluorescent optical microscopy on the right. (C) Fluorescence optical microscopy images of alginate microgels incubated in ethanol.

\section{CONCLUSIONS}

In the present study, an original two-step approach allowing the easy encapsulation of several highly stable oil microdroplets within alginate microgels was developed. Oil microdroplets were 
produced by preparing $\mathrm{O} / \mathrm{W}$ Pickering emulsion, which was stabilized by solid $\mathrm{CNC}$ and $\mathrm{CaCO}_{3}$ particles. It was assessed that even if the emulsification process was made in the presence of a mixture of $\mathrm{CNC}$ and $\mathrm{CaCO}_{3}, \mathrm{CNC}$ were firstly adsorbed onto the oil droplet surface. Oil microdroplet suspension stabilized by a $\mathrm{CNC}$ solid layer, on which $\mathrm{CaCO}_{3}$ particles were adsorbed, was then mixed with an alginate solution to produce alginate microgels containing oil cores using microfluidics. $\mathrm{CaCO}_{3}$ particles co-adsorbed onto the oil microdroplet surface in their inactive form triggered the gelation of alginate microdroplets inside the microfluidic device through in situ internal gelation mechanism. The innovative easy to carry out approach developed in the present study leads to the formation of microgels, based on a biocompatible and biodegradable polymer, which are filled with several oil cores that are efficiently stabilized by bio-based solid interfacial agent. This two-step approach was successfully applied to encapsulate into alginate microgels, oil microdroplets loaded with a model lipophilic compound, namely Nile Red fluorescent dye. It can be thought that the encapsulation of lipophilic compounds within several oil microdroplets stabilized by solid shells should ensure both a better protection and sustained release compared to common encapsulation within only one oil core not stabilized by a solid shell. The double-encapsulation of lipophilic molecules such as vitamins, aroma compounds or anti-cancer drugs within oil microdroplets integrated within naturally-based microgels could be explored in various domains including food, cosmetics and medical applications. 


\section{AUTHOR INFORMATION}

\section{Corresponding Author}

* E-mail: Melanie.Marquis@nantes.inra.fr

\section{Notes}

The authors declare no competing financial interest. 


\section{ACKNOWLEDGMENT}

This work has been funded by the local council program MATIERES. The authors would like to acknowledge Emilie Perrin for her assistance with the preparation of Pickering emulsions. Joëlle Davy and Nicolas Stephan (IMN, Nantes) are acknowledged for SEM microscopy experiments. 


\section{SUPPORTING INFORMATION}

Supporting Information Available: Schematic representation and dimensions of microfluidic flowfocusing device. 


\section{REFERENCES}

(1) McClements, D. J.; Decker, E. A.; Park, Y.; Weiss, J. Designing Food Structure to Control Stability, Digestion, Release and Absorption of Lipophilic Food Components. Food Biophys. 2008, $3,219-228$.

(2) Matalanis, A.; Jones, O. G.; McClements, D. J. Structured Biopolymer-based Delivery Systems for Encapsulation, Protection, and Release of Lipophilic Compounds. Food Hydrocolloids 2011, $25,1865-1880$.

(3) Grant, G. T.; Morris, E. R.; Rees, D. A.; Smith, P. J.; Thom, D. Biological Interactions between Polysaccharides and Divalent Cations: The Egg-box Model. FEBS Lett. 1973, 32, 195-198.

(4) Bugarski, B.; Li, Q; Goosen, M. F. A.; Poncelet, D.; Neufeld, R. J.; Vunjak, G. Electrostatic Droplet Generation: Mechanism of Polymer Droplet Formation. AlChE J. 1994, 40, 1026-1032.

(5) Liu, X. D.; Bao, D. C.; Xue, W. M.; Xiong, Y.; Yun W. T.; Yu, X. J.; Ma, X. J.; Yuan, Q. Preparation of Uniform Calcium Alginate Gel Beads by Membrane Emulsification Coupled with Internal Gelation. J. Appl. Polym. Sci. 2003, 87, 848-852.

(6) Fundueanu, G.; Nastruzzi, C.; Carpov, A.; Desbrieres, J.; Rinaudo, M. Physico-chemical Characterization of Ca-alginate Microparticles Produced with Different Methods. Biomaterials 1999, 20, 1427-1435.

(7) Pritchard, E. M.; Normand, V.; Hu, X.; Budijono, S.; Benczédi, D.; Omenetto, F.; Kaplan, D. L. Encapsulation of Oil in Silk Fibroin Biomaterials. J. Appl. Polym. Sci. 2014, 39990.

(8) Zhang, H.; Tumarkin, E. Sullan, R. M. A.; Walker, G. C.; Kumacheva, E. Exploring Microfluidic Routes to Microgels of Biological Polymers. Macromol. Rapid Commun. 2007, 28, 527-538.

(9) Marquis, M.; Davy, J.; Fang, A.; Renard, D. Microfluidics-ssisted Diffusion Self-assembly: Toward the Control of the Shape and Size of Pectin Hydrogel Microparticles. Biomacromolecules 2014, 15, 1568-1578. 
(10) Zykwinska, A.; Marquis, M.; Sinquin, C.; Cuenot, S.; Colliec-Jouault, S. Assembly of HE800

Exopolysaccharide Produced by a Deep-Sea Hydrothermal Bacterium into Microgels for Protein Delivery Applications. Carbohydr. Polym. 2016, 142, 213-221.

(11) Huang, K. S.; Lai, T. H.; Lin, Y. C. Manipulating the Generation of Ca-alginate Microspheres Using Microfluidic Channels as a Carrier of Gold Nanoparticles. Lab Chip, 2006, 6, 954-957.

(12) Hu, Y. D.; Wang, Q.; Wang, J. Y.; Zhu, J. T.; Wang, H.; Yang, Y. J. Shape Controllable Microgel Particles Prepared by Microfluidic Combining External Ionic Crosslinking. Biomicrofluidics 2012, 6, 026502.

(13) Chen, W. Y.; Kim, J. H.; Zhang, D.; Lee, K. H.; Cangelosi, G. A.; Soelberg, S. D.; Furlong, C. E.; Chung, J. H.; Shen, A. Q. Microfluidic One-Step Synthesis of Alginate Microspheres Immobilized with Antibodies. J. R. Soc., Interface 2013, 10, 20130566.

(14) Choi, C. H.; Jung, J. H.; Rhee, Y. W.; Kim, D. P.; Shim, S. E.; Lee, C. S. Generation of Monodisperse Alginate Microbeads and in situ Encapsulation of Cell in Microfluidic Device. Biomed. Microdev. 2007, 9, 855-862.

(15) Tumarkin, E.; Kumacheva, E. Microfluidic Generation of Microgels from Synthetic and Natural Polymers. Chem. Soc. Rev. 2009, 38, 2161-2168.

(16) Ren, P. W.; Ju, X. J.; Xie, R.; Chu, L. Y. Monodisperse Alginate Microcapsules with Oil Core Generated from a Microfluidic Device. J. Colloid Interface Sci. 2010, 343, 392-395.

(17) Liu, L.; Wu, F.; Ju, X. J.; Xie, R.; Wang, W.; Niu, C. H.; Chu, L. Y. Preparation of Monodisperse Calcium Alginate Microcapsules via Internal Gelation in Microfluidic-generated Double Emulsions. J. Colloid Interface Sci. 2013, 404, 85-90.

(18) Duarte, A. R. C.; Ünal, B.; Mano, J. F.; Reis, R. L.; Jensen, K. F. Microfluidic Production of Perfluorocarbon-Alginate Core-Shell Microparticles for Ultrasound Therapeutic Applications. Langmuir 2014, 30, 12391-12399. 
(19) Um, E.; Nunes, J. K.; Pico, T.; Stone, H. Multicompartment microfibers: fabrication and selective dissolution of composite droplet-in-fiber structures. J. Mater. Chem. B 2014, 2, 78667871.

(20) Pickering, S. U. CXCVI.-Emulsions. J. Chem. Soc., Trans. 1907, 91, 2001-2021.

(21) Xia, Y. N.; Whitesides, G. M. Soft Lithography. Ann. Rev. Mater. Sci. 1998, 28, 153-184.

(22) Kalshnikova, I.; Bizot, H.; Cathala, B.; Capron, I. New Pickering Emulsions Stabilized by Bacterial Cellulose Nanocrystals. Langmuir, 2011, 27, 7471-7479.

(23) Binks, B. P. Particles as surfactants - similarities and differences. Curr. Opin. Colloid Interface Sci. 2002, 7, 21-41.

(24) Arditty, S.; Schmitt, V.; Giermanska-Kahn, J.; Leal-Calderon, F. Materials Based on SolidStabilized Emulsions. J. Colloid Interface Sci. 2004, 275, 659-664.

(25) Du, K.; Glogowski, E.; Emrick, T.; Russell, T. P.; Dinsmore, A. D. Adsorption Energy of Nanoand Microparticles at Liquid-Liquid Interfaces. Langmuir 2010, 26, 12518-12522.

(26) Kalashnikova, I.; Bizot, H.; Cathala, B.; Capron, I. New Pickering Emulsions Stabilized by Bacterial Cellulose Nanocrystals. Langmuir 2011, 27, 7471-7479.

(27) Kalashnikova, I.; Bizot, H.; Cathala, B.; Capron, I. Modulation of Cellulose Nanocrystals Amphiphilic Properties to Stabilize Oil/Water Interface. Biomacromolecules 2012, 13, 267-275.

(28) Kalashnikova, I.; Bizot, H.; Cathala, B.; Capron, I. Cellulose Nanorods of Various Aspect Ratios for Oil in Water Pickering Emulsions. Soft Matter 2013, 9, 952-959.

(29) Revol, J. F.; Bradford, H.; Giasson, J.; Marchessault, R. H.; Gray, D. G. Helicoidal SelfOrdering of Cellulose Microfibrils in Aqueous Suspension. Int. J. Biol. Macromol. 1992, 14, 170172.

(30) Elazzouzi-Hafraoui, S.; Nishiyama, Y.; Putaux, J. L.; Heux, L.; Dubreuil, F.; Rochas, C. The Shape and Size Distribution of Crystalline Nanoparticles Prepared by Acid Hydrolysis of Native Cellulose. Biomacromolecules 2008, 9, 57-65. 
(31) Lehtio, J.; Sugiyama, J.; Gustavsson, M.; Fransson, L.; Linder, M.; Teeri, T. T. The Binding Specificity and Affinity Determinants of Family 1 and Family 3 Cellulose Binding Modules. Proc. Natl. Acad. Sci. U. S. A. 2003, 100, 484-489.

(32) Mazeau, K.; Rivet, A. Wetting the (110) and (100) Surfaces of I $\beta$ Cellulose Studied by Molecular Dynamics. Biomacromolecules 2008, 9, 1352-1354.

(33) Cui, Z. G.; Shi, K. Z.; Cui, Y. Z.; Binks, B. P. Double Phase Inversion of Emulsions Stabilized by a Mixture of $\mathrm{CaCO}_{3}$ Nanoparticles and Sodium Dodecyl Sulphate. Colloids Surf. A 2008, 329, $67-74$.

(34) Zhu, Y; Lu, L.-H.; Gao, J.; Cui, Z.-G.; Binks, B.P. Effect of Trace Impurities in Triglyceride Oils on Phase Inversion of Pickering Emulsions Stabilized by $\mathrm{CaCO}_{3}$ Nanoparticles. Colloids Surf. A 2013, 417, 126-132.

(35) Cristini, V.; Tan, Y. C. Theory and numerical simulation of droplet dynamics in complex flows-a review. Lab Chip, 2004, 4, 257-264.

(36) Garstecki, P.; Fuerstman, M. J.; Stone, H. A.; Whitesides, G. M. Formation of droplets and bubbles in a microfluidic T-junction-scaling and mechanism of break-up. Lab Chip, 2006, 6, 437446.

(37) Greenspan, P.; Mayer, E. P.; Fowler, S. D. Nile Red: a Selective Fluorescent Stain for Intracellular Lipid Droplets. J. Cell Biol. 1985, 100, 965-973.

(38) Malloggi, F.; Thill, A.; Fouilloux, S. Novel method for destabilising a pickering emulsion. Patent WO2013021362 A2, 2013.

(39) Coupland J. N.; Brathwaite D.; Fairley P.; McClements D.J. Effect of Ethanol on the Solubilization of Hydrocarbon Emulsion Droplets in Nonionic Surfactant Micelles. J. Colloid Interface Sci. 1997, 190, 71-75 